\title{
Universal Germline Genetic Testing in Epithelial Ovarian Cancer: Promises and challenges
}

\author{
Rima Sanjay Pathak ${ }^{1}$ Rajiv Sarin ${ }^{1}$ (이 \\ ${ }^{1}$ Department of Radiation Oncology, Tata Memorial Centre, Homi \\ Bhabha National Institute, Mumbai, Maharashtra, India \\ Ind J Med Paediatr Oncol 2022;43:193-194.
}

Address for correspondence Rima Sanjay Pathak, MD, 1128, Homi Bhabha Block, Department of Radiation Oncology, Tata Memorial Hospital, Dr. Earnest Borges Road, Parel, Mumbai 400012, Maharashtra, India (e-mail: drrimapathak@gmail.com).

Moreover, the frequency and spectrum of BRCA1/2 and other gene mutations vary in different geoethnic groups. ${ }^{2,3,9}$ The frequency and the type of mutations associated with various histologies also differ. ${ }^{9}$

A study from Ontario evaluating physician practices showed that genetic testing rates and referrals reduce with the increasing complexity of testing criteria. ${ }^{10}$ Research studies employing germline multigene panel testing of large unselected EOC populations could provide a more reliable estimate of the prevalence of germline mutations and the most commonly mutated genes in EOC cases in the population. This information would facilitate genetic counseling and help frame evidence-based guidelines for genetic testing, which is an expensive test with a turn-around time of 4 to 6 weeks.

This study provides the much-required detailed information of the prevalence in unselected Indian ovarian cancer patient population (15.5\%) while also providing estimates for various enriched subgroups of patients who are $\leq 50$ years (22.2\%), serous epithelial ovarian cancers $(25.2 \%)$, or those with a family history of cancer (55.6\%). ${ }^{11}$ The results show that the prevalence of germline mutations is much higher than most solid tumors. Contrary to expectations, subgroups such as non-serous (13.8\%), older than 50 years $(20.8 \%)$, or those without a family history (20.2\%) also had a clinically relevant prevalence of germline mutation. ${ }^{11}$ This implies that several patients will be unable to qualify for the genetic test if any selection criteria are used. Identifying the maximum number of index EOC cases will not only help to personalize their therapy, provide cancer surveillance, and ensure future cancer prevention but also extend the benefit of identifying family members who have inherited the mutation and through culturally sensitive and evidence-

(c) 2022. Indian Society of Medical and Paediatric Oncology. All rights reserved.

This is an open access article published by Thieme under the terms of the Creative Commons Attribution-NonDerivative-NonCommercial-License, permitting copying and reproduction so long as the original work is given appropriate credit. Contents may not be used for commercial purposes, or adapted, remixed, transformed or built upon. (https://creativecommons.org/ licenses/by-nc-nd/4.0/)

Thieme Medical and Scientific Publishers Pvt. Ltd., A-12, 2nd Floor, Sector 2, Noida-201301 UP, India 
based genetic counseling, testing, and risk management approaches. $^{12,13}$ The recent negative results of the UK Collaborative Trial of Ovarian Cancer Screening study of lack of benefit of ovarian cancer screening using annual transvaginal ultrasound or longitudinal CA- $125^{14}$ highlights the continued relevance of timely risk-reducing salpingo-oopheorectomy for women with germline BRCA1/2 mutations.

With patients enrolled from three centers in Delhi, two in Mumbai, and one each in Bengaluru and Hyderabad, major geoethnic groups within India are likely to be represented. However, with a relatively small cohort of 239 cases and the non-inclusion of several large and small Indian states, several geoethnic groups with a different population prevalence of germline BRCA mutations and founder mutations would be under-represented. Furthermore, it is unclear if this represents a consecutive patient cohort or some form of selection was present. As seen previously in many studies that selecting patients based on any criteria can inflate the prevalence leading to erroneous estimates. In addition to the BRCA1/2 genes, mutations in other genes associated with the development of ovarian cancer such as PALB2, PRIB1, the core RAD genes, CHEK2 among others were not reported even though a 94 gene NGS panel was used. ${ }^{2,7,11}$ Moreover, copy number variation or large genomic rearrangement were not reported, which potentially lead to underestimation of the prevalence.

Are we ready to implement genetic counseling and testing in unselected EOC population? As per the GLOBOCAN 2018 data, more than 36,000 ovarian cancer patients are diagnosed every year in India, with the vast majority being EOC. ${ }^{1}$ Currently, in India, there are a limited number of trained genetic counselors and pre-test genetic counseling will be the rate-limiting step if unselected population testing is implemented. Therefore, before the implementation of unselected population testing, building the required capacity and infrastructure by adopting the mainstreaming pathway is required. Here, the primary care physician/oncologist provides pre-test counseling for patients without a significant family history or psychosocial issues and detailed posttest counseling of mutation carriers in the cancer genetics clinic. Such a model has been adopted in the UK and Malaysia and has been shown to improve access to genetic testing by reducing waiting times for counseling. ${ }^{15}$

Thus, this study provides important information on the prevalence of germline mutations among ovarian cancer patients from India and leads to the realization of many challenges with its practical implementation across the country. There is a need for such studies to generate the prevalence of germline mutations in other cancer-predisposing genes in ovarian cancers of different histologies and other common cancers among Indian patients. This is among the first few steps toward the expansion of access to genetic testing among cancer patients and their family members in India.

\section{Funding}

None.

\section{Conflict of Interest}

Dr. Rima Sanjay Pathak is a member on the Data safety and monitoring at Tata Memorial Centre, Mumbai.

\section{References}

1 Bray F, Ferlay J, Soerjomataram I, Siegel RL, Torre LA, Jemal A. Global cancer statistics 2018: GLOBOCAN estimates of incidence and mortality worldwide for 36 cancers in 185 countries. CA Cancer J Clin 2018;68(06):394-424

2 Li W, Shao D, Li L, et al. Germline and somatic mutations of multigene panel in Chinese patients with epithelial ovarian cancer: a prospective cohort study. J Ovarian Res 2019;12; article no. 80 Doi: 10.1186/s13048-019-0560-y

3 Kast K, Rhiem K, Wappenschmidt B, et al; German Consortium for Hereditary Breast and Ovarian Cancer (GC-HBOC) Prevalence of BRCA1/2 germline mutations in 21401 families with breast and ovarian cancer. J Med Genet 2016;53(07):465-471

4 Singh J, Thota N, Singh S, et al. Screening of over 1000 Indian patients with breast and/or ovarian cancer with a multi-gene panel: prevalence of BRCA1/2 and non-BRCA mutations. Breast Cancer Res Treat 2018;170(01):189-196

5 Daly MB, Pilarski R, Yurgelun MB, et al. NCCN guidelines insights: genetic/familial high-risk assessment: breast, ovarian, and pancreatic, version 1.2020. J Natl Compr Canc Netw 2020;18(04): 380-391

6 Konstantinopoulos PA, Norquist B, Lacchetti C, et al. Germline and somatic tumor testing in epithelial ovarian cancer: ASCO guideline. J Clin Oncol 2020;38(11):1222-1245

7 Walsh T, Casadei S, Lee MK, et al. Mutations in 12 genes for inherited ovarian, fallopian tube, and peritoneal carcinoma identified by massively parallel sequencing. Proc Natl Acad Sci U S A 2011;108(44):18032-18037

8 Dos Santos Vidal R, Hawrysh A, Walia JS, Davey S, Feilotter H. Eligibility criteria and genetic testing results from a high-risk cohort for hereditary breast and ovarian cancer syndrome in southeastern Ontario. J Mol Diagn 2016;18(03):362-369

9 Sugino K, Tamura R, Nakaoka H, et al. Germline and somatic mutations of homologous recombination-associated genes in Japanese ovarian cancer patients. Sci Rep 2019;9:17808

10 Myers MF, Chang MH, Jorgensen C, et al. Genetic testing for susceptibility to breast and ovarian cancer: evaluating the impact of a direct-to-consumer marketing campaign on physicians' knowledge and practices. Genet Med 2006;8(06):361-370

11 Gupta S, Rajappa S, Advani S, et al. Prevalence of BRCA1 and BRCA2 mutations among patients with ovarian, primary peritoneal, and Fallopian tube cancer in India: a multicenter cross-sectional study. JCO Glob Oncol 2021;7(07):849-861

12 Lambert M. ACOG guidelines for managing hereditary breast and ovarian cancer syndrome. Am Fam Physician 2009;80(12): 1505-1507

13 Paluch-Shimon S, Cardoso F, Sessa C, et al; ESMO Guidelines Committee. Prevention and screening in BRCA mutation carriers and other breast/ovarian hereditary cancer syndromes: ESMO clinical practice guidelines for cancer prevention and screening. Ann Oncol 2016;27(Suppl 5):v103-v110

14 Menon U, Gentry-Maharaj A, Burnell M, et al. Ovarian cancer population screening and mortality after long-term follow-up in the UK Collaborative Trial of Ovarian Cancer Screening (UKCTOCS): a randomised controlled trial. Lancet 2021;397 (10290):2182-2193|

15 Kemp Z, Turnbull A, Yost S, et al. Evaluation of cancer-based criteria for use in mainstream BRCA1 and BRCA2 genetic testing in patients with breast cancer. JAMA Netw Open 2019;2(05): e194428 